\title{
ELETROCOAGULAÇÃO EM SISTEMA CONTÍNUO PARA O TRATAMENTO DE ÁGUAS RESIDUAIS DA SUINOCULTURA SUBMETIDAS À PRÉ-DIGESTÃO ANAERÓBIA
}

\author{
R. MORES ${ }^{1}$, R. M. DALLAGO ${ }^{1}$, J. STEFFENS ${ }^{1}$, A. KUNZ ${ }^{2}$, A. ANTUNES ${ }^{1}$ e V. ADAM ${ }^{1}$ \\ ${ }^{1}$ Universidade Regional Integrada do Alto Uruguai e das Missões, \\ Departamento de Engenharia de Alimentos \\ ${ }^{2}$ Embrapa Suínos e Aves \\ E-mail para contato: rubiamores@yahoo.com.br
}

\begin{abstract}
RESUMO - A água residual da suinocultura tratada por biodigestor apresenta elevado potencial poluidor podendo ocasionar impactos ambientais indesejáveis, quando manejada de maneira incorreta. Neste contexto, a eletrocoagulação (EC) foi estudada como alternativa de tratamento dessas águas residuais, em sistema contínuo. O efluente utilizado no tratamento foi coletado na saída de um biodigestor na unidade da Embrapa Suínos e Aves. O reator de EC em fluxo contínuo possui capacidade de tratar 1,480 litros de efluente. Os parâmetros estudados foram: a voltagem $(5,0 \mathrm{~V})$, distância entre os eletrodos $(2 \mathrm{~cm})$, Tempo de Residência Hidráulico (60 min.) e o pH $(6,0)$. Os resultados de eficiência de remoção (\%) de cor, turbidez, COT, DQO, Ntotal e ST foram de 96,2\%, 96,3\%, $81,8 \%, 82,9 \%, 17,9 \%$ e $60,4 \%$ respectivamente. Sendo assim, o sistema de EC em fluxo contínuo reproduziu resultados satisfatórios de remoção, assim tornando-se uma alternativa tecnologia promissora a ser avaliada.
\end{abstract}

\section{INTRODUÇÃO}

Em 2013, o rebanho médio de suíno no Brasil foi 38.578 milhões de animais, fazendo do Brasil o quarto maior produtor e exportador de carne suína do mundo (USDA, 2013). Assim, a suinocultura representa um dos maiores segmentos de produção animal do Brasil, sendo seu crescimento impulsionado pela demanda de carne suína no mundo.

De acordo BROETTO et al. (2014), a expansão acelerada da produção de suínos no Brasil tem aumentado a geração de resíduos líquidos ricos em matéria orgânica, nutrientes e metais com alto potencial de poluição do ar, água e solos (STEINMETZ et al., 2009).

O tratamento por digestão anaeróbia é cada vez mais difundido como uma forma de tratar águas residuais da suinocultura, uma vez que produz energia renovável (metano), e valiosos resíduos biodegradáveis. No entanto, o sistema ainda enfrenta algumas limitações vitais ao seu bom funcionamento, proporcionando uma perda de eficiência do biodigestor, assim, o biofertilizante não pode ser utilizado ou descartado diretamente nos solo e nos corpos d'água, pois ainda apresenta alto potencial poluidor (KUNZ et al., 2005).

Desta forma, a eletrocoagulação (EC) surge como alternativa de tratamento dos resíduos da digestão anaeróbia. No processo EC, o coagulante é gerado in situ por oxidação eletrolítica 
do ânodo (Al e/ou Fe). A EC envolve três etapas sucessivas: (a) formação do agente coagulante por oxidação eletrolítica; (b) desestabilização dos contaminantes em partículas em suspensão e quebra das emulsões; (c) a agregação das fases que se desestabilizaram em forma de flocos (MOLLAH et al., 2001). No cátodo ocorre a hidrólise da água, os gases gerados são responsáveis pela flotação dos flocos formados. As principais reações que ocorrem no ânodo e no cátodo durante o processo de tratamento por EC quando se utiliza eletrodos de Al estão descritos nas Equações 1 e 2 . Os íons $\mathrm{Al}^{+3}$ liberados pelo ânodo em $\mathrm{pH}$ próximo ao neutro o formam $\mathrm{Al}(\mathrm{OH})_{3}$.

Ânodo:

$$
A l_{(s)} \rightarrow A l_{(a q)}^{+3}+3_{-}^{-}
$$

Cátodo:

$$
2 \mathrm{H}_{2} \mathrm{O}+2_{\bar{e}} \rightarrow \mathrm{H}_{2(g)}+2 \mathrm{OH}_{(a q)}
$$

Portanto, o objetivo desse estudo foi avaliar a EC em fluxo contínuo como tratamento de águas residuais da suinocultura tratadas por digestão anaeróbia, utilizando eletrodos de alumínio.

\section{MATERIAIS E MÉTODOS}

O efluente utilizado no estudo foi coletado da saída do biodigestor de fibra, com fluxo ascendente, de volume útil de $10 \mathrm{~m}^{3}$, com vazão de alimentação de $15 \mathrm{~L} / \mathrm{h}$ de dejeto e tempo de retenção hidráulica de 28 dias, na Estação de Tratamento de Dejetos Suínos da Embrapa Suínos e Aves, localizada em Concórdia, oeste de Santa Catarina. O efluente foi coletado em galões de polietileno de $5 \mathrm{~L}$ e armazenados a temperatura de $-10{ }^{\circ} \mathrm{C}$, com a finalidade de conservar suas características.

Para realização dos ensaios eletroquímicos foi constituído de um reator de fluxo contínuo confeccionado em vidro, que possuía um volume útil de $1,480 \mathrm{~L}$, dois pares de eletrodos de alumínio com dimensões $150 \mathrm{~mm} \times 70 \mathrm{~mm} \times 2 \mathrm{~mm}$, que foram inseridos verticalmente e conectados a uma fonte de corrente contínua (MIT DC Power Supply MS 3005). Os eletrodos de alumínio foram separados por um suporte confeccionado em vidro com dimensões de $170 \mathrm{~mm}$ x $20 \mathrm{~mm}$ x $2 \mathrm{~mm}$ (Figura 1). As condições operacionais mantidas constantes foram: a voltagem 5,0 Volts (V), distância entre os eletrodos (DE) $2 \mathrm{~cm}$, Tempo de Residência Hidráulico (TRH) 60 min. e pH 6,0 (ajustado com $\mathrm{H}_{2} \mathrm{SO}_{4}$ ).O experimento foi realizado em triplicata. 
Figura 1 - Módulo experimental

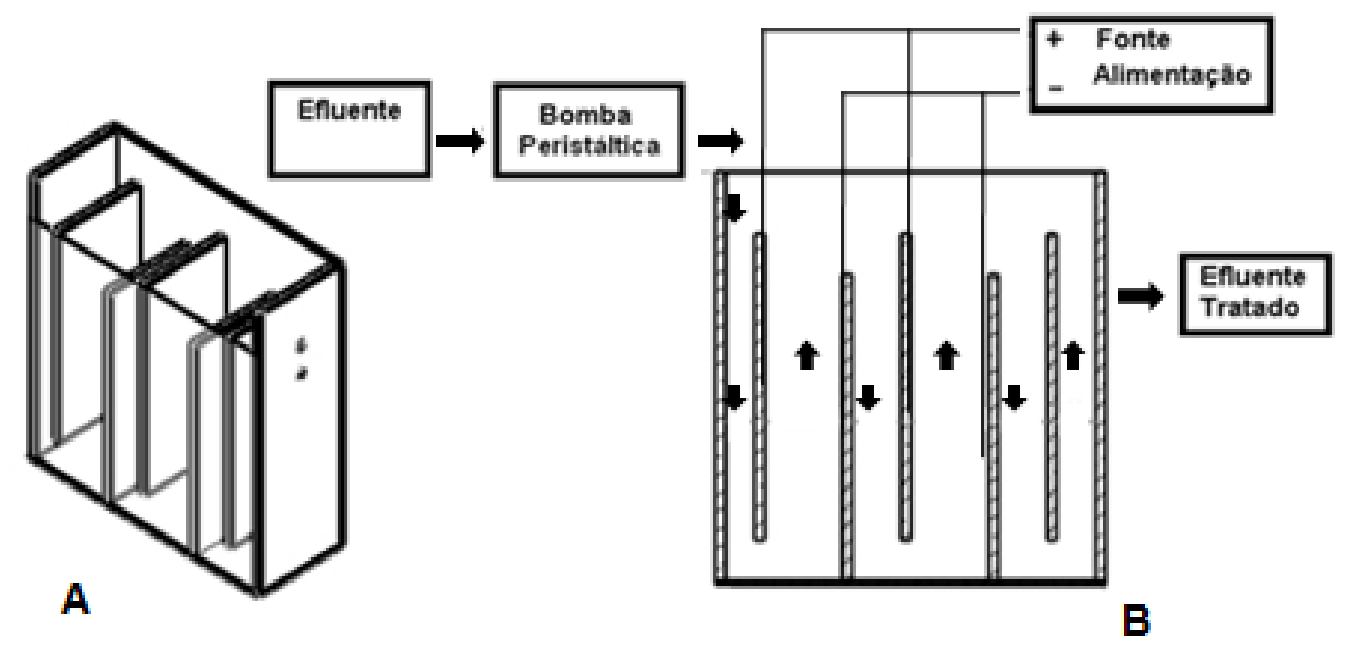

A eficiência do processo foi acompanhada mediante a retirada de $10 \mathrm{~mL}$ do efluente tratado a cada 10 minutos por um período de 240 minutos. As seguintes respostas foram acompanhadas: cor, turbidez, carbono orgânico total (COT), demanda química de oxigênio (DQO), $\mathrm{pH}$, e densidade de corrente. A cor e a turbidez foram mensuradas através do método espectrofotométrico, utilizando o colorímetro o Hach (DR870). $\mathrm{O} \mathrm{pH}$ foi medido usando o pHmetro da Metrhm ( $\mathrm{pH}$ Lab 837). A condutividade foi medida em um condutivímetro portátil Oakton (Con 10 meter). Os níveis de COT foram analisados segundo a metodologia padrão ISO-1987 em equipamento Shimadzu (TOC-5000A). A DQO foi determinada usando o método colorimétrico em microescala (5220D, APHA, 1992), onde as amostras foram preparadas e conduzidas para digestão em um termoreator Marconi (Dry Block MA 4004), após a digestão realizou-se as leituras no colorímetro digital Hach (DR870).

\section{RESULTADOS E DISCUSSÃO}

A Tabela 1 apresenta a caracterização do efluente proveniente da digestão anaeróbia de afluentes da suinocultura e a caracterização do efluente após o tratamento por EC em valores reais e em percentagem de remoção.

Tabela 1 - Caracterização do afluente bruto e efluente tratado.

\begin{tabular}{cccc}
\hline Parâmetro & Efluente Bruto & $\begin{array}{c}\text { Efluente Tratado } \\
120 \text { minutos }\end{array}$ & $\begin{array}{c}\text { Eficiência } \\
(\%)\end{array}$ \\
\hline Cor Aparente $\left(\mathrm{mg}_{\mathrm{PtCo}} / \mathrm{L}\right)$ & $69.866,7 \pm 31.830,4$ & $2.333,3 \pm 548,5$ & 96,2 \\
Turbidez $(\mathrm{NTU})$ & $9.200,0 \pm 4.660,4$ & $291,7 \pm 62,9$ & 96,3 \\
COT $(\mathrm{mg} / \mathrm{L})$ & $2.314,0 \pm 291,4$ & $417,3 \pm 51,1$ & 81,8 \\
DQO $(\mathrm{mg} / \mathrm{L})$ & $8.603,3 \pm 2.820,7$ & $1.631,3 \pm 943,7$ & 82,9 \\
N-Total $(\mathrm{mg} / \mathrm{L})$ & $1.693,7 \pm 68,4$ & $1.386,2 \pm 88,4$ & 17,9 \\
ST $(\mathrm{mg} / \mathrm{L})$ & $19.966,7 \pm 745,7$ & $7.883,3 \pm 1.260,6$ & 60,4 \\
\hline
\end{tabular}


Para a condição de tratamento aplicada neste estudo (5,0 V, DE de $2 \mathrm{~cm}$ e o TRH de 60 min.), remoções de $96,2 \%, 96,3 \%, 81,8 \%, 82,9 \%, 17,9 \%$ e $60,4 \%$ foram alcançadas para as respostas cor, turbidez, COT, DQO, Ntotal e ST, respectivamente. CHO et al. (2010), em seu estudo com eletrodos de Ti revestidos com óxido de irídio, com um TRH de 6 h, obteve 99\% de remoção de $\mathrm{NH}_{4}, 94 \%$ para o nitrogênio solúvel, $64 \%$ de remoção de carbono orgânico solúvel e $93 \%$ de remoção da cor para o tratamento de lixiviados do sistema de filtragem biológica da compostagem de dejetos suínos.

Para melhor visualização do comportamento das respostas cor, turbidez, N-Total e COT, durantes os 240 minutos de tratamento a Figura 2 mostra evolução das respostas acompanhadas, em percentagem de remoção de em função do tempo operacional.

Para todas as respostas acompanhadas observa-se um aumento da eficiência até $\cong 120$ minutos operacionais, assim atingindo um regime estacionário de remoção e mantendo-se até os 240 minutos.

Figura 2 - Remoção dos parâmetros da cor, DQO, turbidez, COT e $\mathrm{N}_{\text {Total }}$ em função do tempo operacional do fluxo contínuo



De acordo com Adhoum et al. (2004), no reator de EC a densidade de corrente é um dos parâmetros mais importantes para controlar a taxa de reação nos processos eletroquímicos. Ela determina a taxa de produção de coagulante, bem como o ajuste da produção e tamanho de bolhas, afetando assim a formação dos flocos e o processo de separação por flotação (KOBYA et al., 2008). A Figura 3 mostra a variação da DC no decorrer dos 240 minutos de tratamento. Observa-se que a DC após os 60 minutos de tratamento a mesma apresentou pouca variação. 
Figura 3 - Variação da densidade da corrente em função do tempo operacional



$\mathrm{Na}$ Figura 4, pode ser visto o monitoramento do $\mathrm{pH}$ em função do tempo de tratamento. $\mathrm{O}$ tratamento por EC é altamente dependente do $\mathrm{pH}$ do afluente, pois o $\mathrm{pH}$ (6-8) é um parâmetro importante para a formação do $\mathrm{Al}(\mathrm{OH})_{3}$, responsável pela formação dos flocos (MOLLAH et al., 2004).

Figura 4 - Variação do pHs em função do tempo operacional

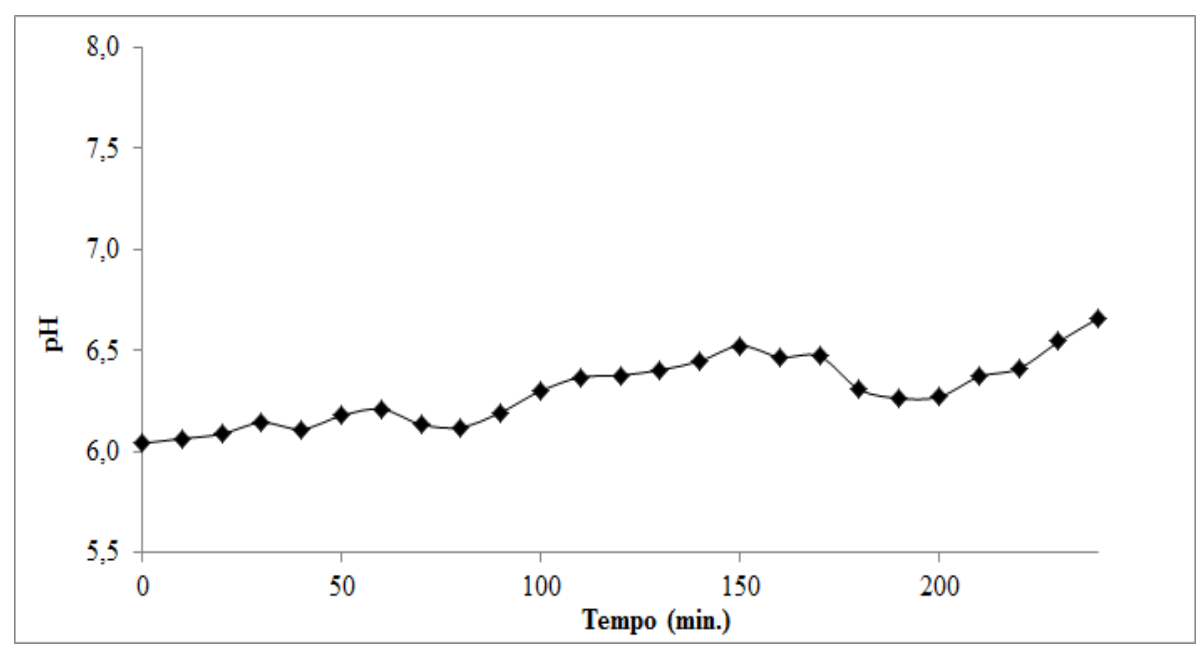

Observa-se que o pH mantém-se dentro da faixa ideal de formação do hidróxido de alumínio, assim pode-se dizer que os valores de $\mathrm{pH}$ estão vinculados com a estabilidade de remoção das respostas acompanhadas. 


\section{CONCLUSÕES}

A eletrocoagulação em sistema de fluxo contínuo mostrou-se eficiente para a remoção de cor, turbidez, COT e DQO, apresentando remoções acima de $80 \%$. Portanto, o tratamento do efluente de biodigestão anaeróbia de afluentes da suinocultura por eletrocoagulação mostra-se promissora.

\section{AGRADECIMENTOS}

URI -Erechim, CAPES, Embrapa Suínos e Aves e CNPq.

\section{REFERENCIAS}

ADHOUM, N.; MONSER, L.; BELLAKHAL, N.; BELGAIED, J. Treatment of electroplating wastewater containing $\mathrm{Cu}^{2+}, \mathrm{Zn}^{2+}$ and $\mathrm{Cr}(\mathrm{VI})$ by electrocoagulation. $J$. Hazard. Mater., v. B112, p. 207-213, 2004.

BROETTO, T.; TORNQUIST, C. G.; BAYER, C.; CAMPOS, B. C.; MERTEN, C. G.; WOTTRICH, B. Soils and surface waters as affected by long-term swine slurry application in oxisols of southern Brazil. Pedosphere, v. 24, p. 585-594, 2014.

CHO, J.H.; LEE, J.E.; RA, C.S.. Effects of electric voltage and sodium chloride level on electrolysis of swine Wastewater. J. Hazard. Mater., v. 180, p. 535-541, 2010.

KOBYA, M.; DELIPINAR, S.. Treatment of the baker's yeast wastewater by electrocoagulation, J. Hazard. Mater., v. 154, p. 1133-1140, 2008.

KUNZ, A.; HIGARASHI, M. M.; OLIVEIRA, P. A. Tecnologias de manejo e tratamento de dejetos de suínos estudadas no Brasil. Caderno de Ciência e Tecnologia, v. 22, (3), p. 652-665, 2005.

MOLlAH, M. Y. A.; MORKOVSKY, P.; GOMES, J. A. G.; KESMEZ, M.; PARGA, J.; COCKE, D. L.. Fundamentals, present and future perspectives of electrocoagulation. $J$. Hazard. Mater., v. B114, p. 199-210, 2004.

MOLLAH, M. Y. A.; SCHENNACH, R.; PARGA, J. R.; COCKE, D. L.. Electrocoagulation (EC)—science and applications. J. Hazard. Mater., v. B84, p. 29-41, 2001.

STEINMETZ, R. L. R.; KUNZ, A.; DRESSLER, F. E. M. M.; MARTINS, A. F. Study of metal distribution in raw end screened swine manure. CLEAN-Soil, Air, Water, v. 37 (3), p. 239-244, 2009.

USDA, 2013. USDA Livestock and Poultry: World Markets and Trade. 2014: Record Global Meat Trade. United States Department of Agriculture. Foreign Agricultural Service (2013) 